\title{
Über das Genus Inocybe in Finnland. Die neuen Arten I. mytiliodora und I. urceolicystis
}

\author{
JOHANN STANGL und JUKKA VAURAS
}

STANGL, J. \& VAURAS, J. 1988: Finnish records on the genus Inocybe. The new species I. mytiliodora and I. urceolicystis. - Karstenia 27: 15-21.

Two new species, Inocybe mytiliodora Stangl \& Vauras and Inocybe urceolicystis Stangl \& Vauras, are described from Finland. Both are smooth-spored and have metuloid cystidia. I. mytiliodora is characterized by a smell reminiscent that of Mytilus, and short, broad and thin-walled hymenium cystidia. The stipe of this species is not pruinose. The best differentiating character of $I$. urceolicystis is found in the microscopy: the cystidia are nearly unique to the smooth-spored species in the genus in being rounded basally. That species has pruinose stipe apex and spermatic smell.

Both rare species are described and discussed, and the descriptions are completed with photographs, and habitus and microscopical drawings. Inocybe appendiculata Kühner and I. nematoloma Joss. are reported as new to Finland.

STANGL, J. \& VAURAS, J. 1988: Über das Genus Inocybe in Finnland. Die neuen Arten I. mytiliodora und I. urceolicystis. - Karstenia 27: 15-21.

Es werden zwei neue Risspilze, Inocybe mytiliodora Stang1 \& Vauras und Inocybe urceolicystis Stangl \& Vauras, aus Finnland beschreiben. Beide Arten haben glattwandige Sporen und metuloide Zystiden. Charakteristisch für I. mytiliodora sind der Geruch wie in Mytilus und die kurzen, breiten, dünnwandigen Hymenialzystiden. Der Stiel dieser Art ist nicht bereift. Das beste Merkmal der Art I. urceolicystis ist microskopisch: die Zystiden sind fast unvergleichlich bei den glattsporigen Risspilzarten wegen der abgerundeten Basis. Der Stiel dieser Art ist oben bereift, und der Geruch ist spermatisch.

Beide seltene Arten werden beschrieben und diskutiert. Die Beschreibungen werden durch Photographien, Habitus- und Mikrozeichnungen ergänzt. Die Erstfunde, in Finnland, von Inocybe appendiculata Kühner und I. nematoloma Joss. werden gemeldet.

Johann Stangl, Von-der-Tann-Strasse 48, D-8900 Augsburg, Bundesrepublik Deutschland

Jukka Vauras, Biologisches Institut, Universität Turku, SF-20500 Turku, Finnland 

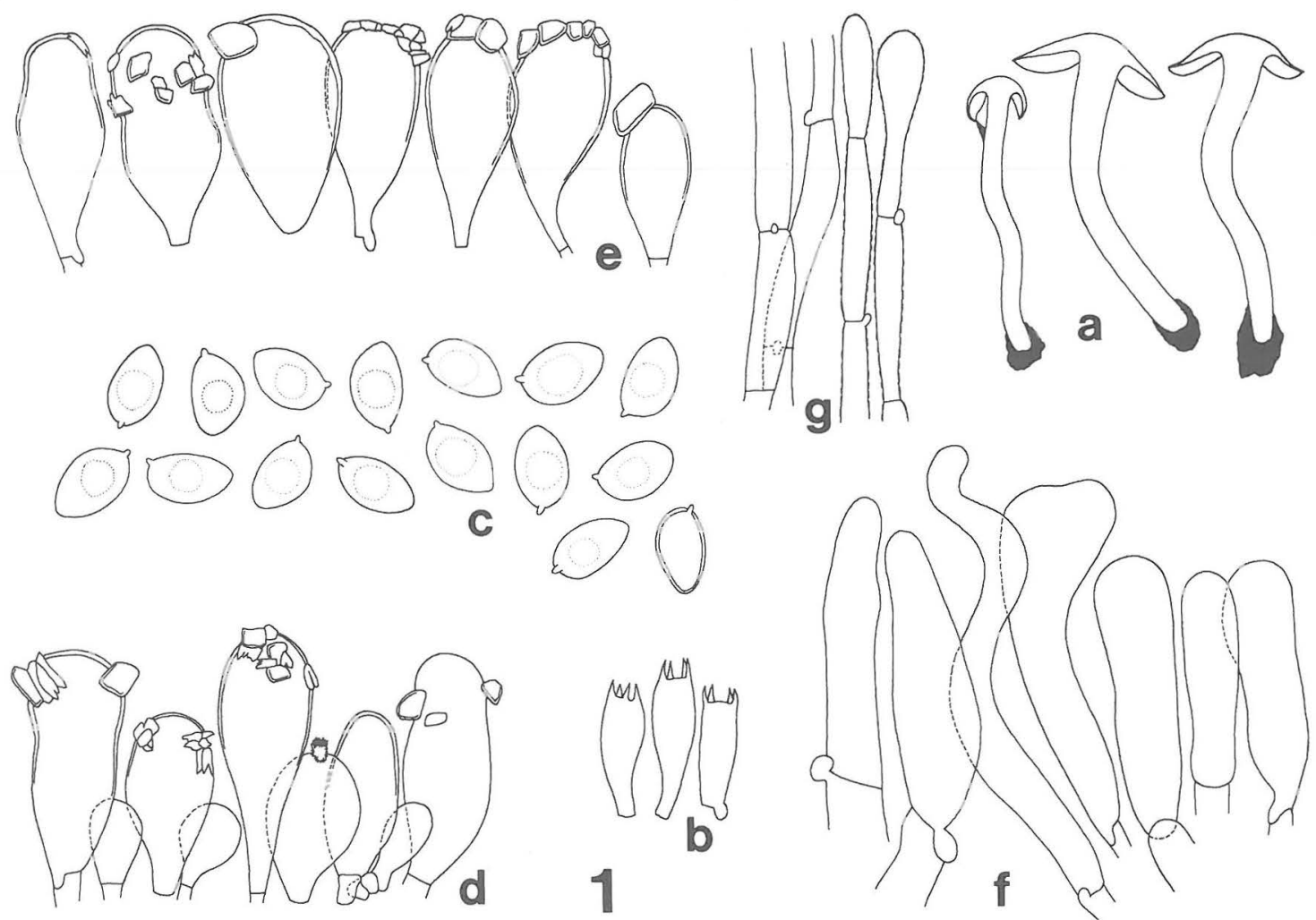

Abb. 1. Inocybe mytiliodora (Holotypus). a) Fruchtkörper, b) Basidien, c) Sporen, d) Cheilozystiden, e) Pleurozystiden, f) Hyphen der Stielbekleidung, oben, g) Hyphen der Hutbedeckung. Skala: Fruchtkörper x 1, Sporen x 1000, die andere x 500 .

Inocybe mytiliodora Stangl \& Vauras sp. nov. -Abb. 1, 3

Pileus 0.4-2 cm latus, $0.4-1.1 \mathrm{~cm}$ altus, primum semiglobosus, demum campanulato-convexus, umbone obtuso, mox squamoso adpresso vel multum fatiscente, margine longe incurvato, paulum in fibras radiales fatiscente, colore pileopallide rubro-brunneo velochraceo-brunneo. Cortinapallida. Lamellaepotius confertae, usque ad $2 \mathrm{~mm}$ latae, adnexae, primum pallidae mox brunneae, acie pallidae, subtiliter fimbriatae. Stipes 2-4 cm longus, $1.5-4 \mathrm{~mm}$ crassus, cylindraceus sed interdum apicem vel basem versus leviter incrassatus, curvus, ad apicem albo-fibrillosoflocculosus et subalbus, basem versus fibris crassis striatus et pileo concolorosus. Contextus pilei et stipitis albus, ad marginem stipitis pallide luteus, odore peculiari, paulum sicut molluscorum foetoris simili.

Sporae (7.5-)8.5-10(-10.5) x (5-)5.5-6.5 $\mu \mathrm{m}$, ovales vel subamygdaliformes. Cystidia hymenii 28 $57 \times 14-27 \mu \mathrm{m}$, brevia, lata, saepe cum magnis crys- tallis instructa, membranis tenuibus, sine collo, usque ad 1 um crassis, in $\mathrm{NH}_{4} \mathrm{OH}$ hyalinis vel dilute luteis. Metuloida ad apicem stipitis desunt. Habitatio: ad terram calcaream congregatim sub arboribus frondosis (Quercubus, Betulis).

Typus: Finnland. Varsinais-Suomi: Parainen, Malmnäs, in der Nähe von einem Kalkwerk, in Park unter Quercus robur, in der Nähe auch Betula pendula, auf Lehmboden, oft zwei Fruchtkörper beisammen wachsend, ca 25 Fruchtkörper in Gesellschaft von Achillea millefolium, Carex digitata, Lathyrus pratensis, Listera ovata, Pimpinella saxifraga, Trifolium pratense, T. repens, Entoloma undatum (Fr.) Mos. (ss. Mos.), Helvella crispa Fr., Inocybe amblyspora Kühner, Peziza succosa Berk. und Pluteus exiguus (Pat.) Sacc., Grid $27^{\circ} \mathrm{E}:$ 66961:2400, Höhe 15 m, 29.VII.1985 J. Vauras 1876F (TUR, Holotypus; H, L, M, WTU, Isotypi).

Etymologie: mytiliodorus, der Geruch ist wie in Muscheln (Mytilus edulis). 

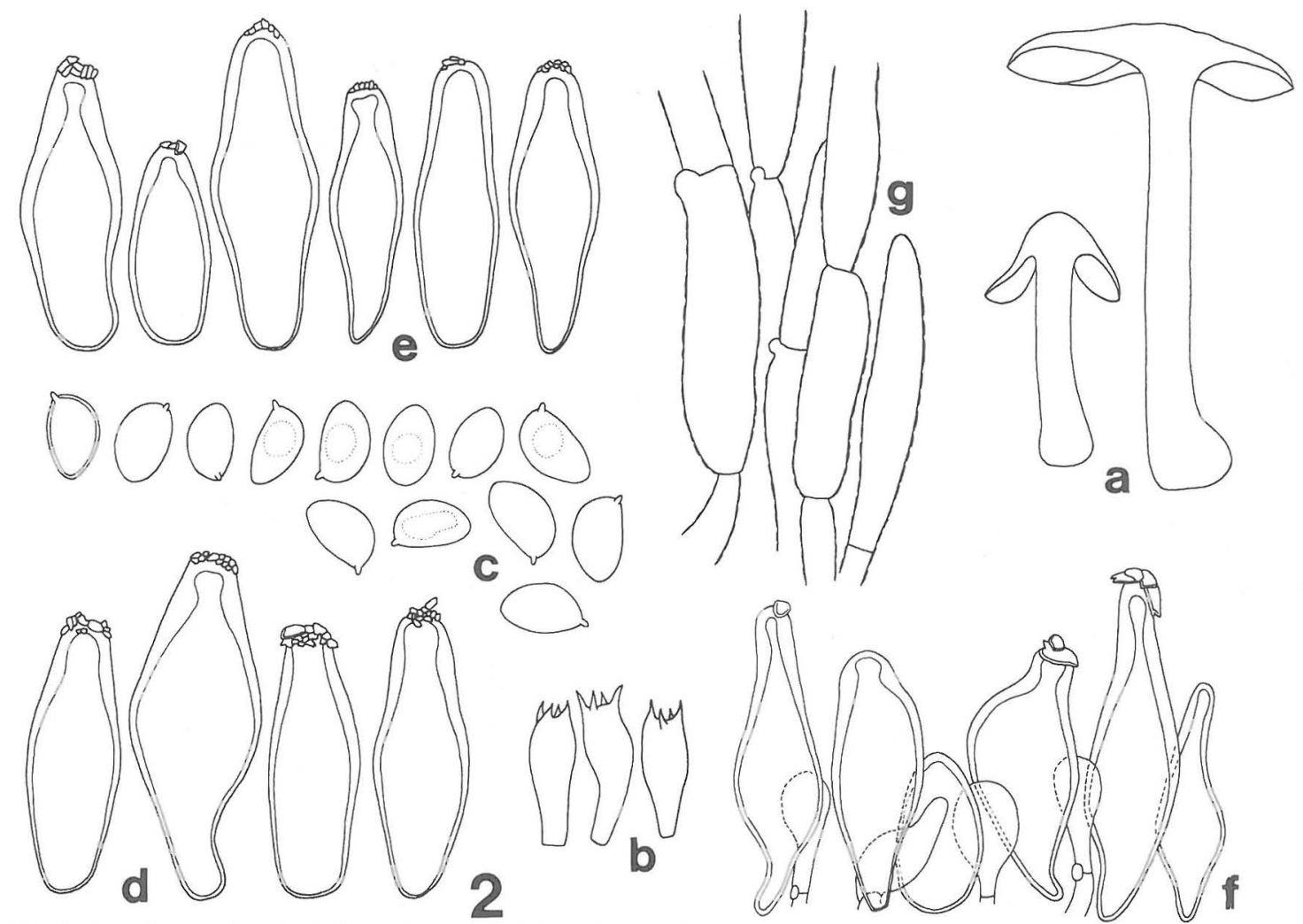

Abb. 2. Inocybe urceolicystis (Holotypus). a) Fruchtkörper, b) Basidien, c) Sporen, d) Cheilozystiden, e) Pleurozystiden, f) Kaulozystiden, oben, g) Hyphen der Hutbedeckung. Skala: wie Abb. 1.

Hut $0.4-2 \mathrm{~cm}$ breit und $0.4-1.1 \mathrm{~cm}$ hoch; jung halbkugelig, dann kegelig gewölbt, alt fast scheibenförmig, mit kaum vorgezogenem abgerundetem Scheitel, Rand oft etwas aufgebogen, aber lange ganz aussen eingebogen bleibend; Hutbedeckung schon jung ungleichmässig, auf der Scheibe spaltend, Rand manchmal grobfaserig, mit Lupe etwas faserschuppig, matt; rotbraun, gelbbraun, lederbraun (Cailleux 69/M, 75-77/M) (als Exsiccate 67/N); nicht hygrophan. Cortina weisslich. Lamellen ziemlich dicht stehend, untermischt, bis $2 \mathrm{~mm}$ breit, \pm halb angewachsen; jung graulich, dann sandfarben (90/L), dann hellbraun $(69 / \mathrm{N}$ ), alt braun (75/P) (als Exsiccate zunächst 60/P aber dunkler); Schneide hell, bewimpert. Stiel $2-4 \mathrm{~cm}$ hoch, $1.5-4 \mathrm{~mm}$ breit; zylindrisch, \pm gleichdick, immer gewunden; wie Hut gefärbt, an der Spitze weisslich und feinbeschürfelt (aber nicht mit Metuloiden), zur Basis hin braunfaserig, oft zonenweise einreissend. Fleisch weiss, am Stielrand hyalin gelblich. Geruch unangenehm, etwas wie in Muscheln (Mytilus edulis), wie Konservenbüsche mit gesalzenen Muscheln im Wasser (dieser Muschelgeruch war noch an einem ein Jahr alten Exsiccat wahrnehmbar), nicht spermatisch.

Sporen (im Stielpräparat gemessen) (7.5-)8.5$10(-10.5) \times(5-) 5.5-6.5 \mu \mathrm{m}$, Mittelwert $8.9 \times 5.7$ $\mu \mathrm{m}, \mathrm{Q}=(1.3-) 1.5-1.6(-1.8), \overline{\mathrm{Q}}=1.6$, glatt, oval bis \pm mandelförmig. Basidien $24-30 \times 8-10 \mu \mathrm{m}$, vorwiegend mit vier Sterigmen. Hymenialzystiden 28$57 \times 14-27 \mu \mathrm{m}$, kurz, breit, blasig, auffällig dünnwandig. Metuloide Kaulozystiden keine. Stielbekleidung an der Spitze aus 7-21 $\mu \mathrm{m}$ dicken Hyphen bestehend. Hyphen der Hutbedeckung 6-13 $\mu \mathrm{m}$ dick, schwach angelagert inkrustiert.

Inocybe mytiliodora ist aus der hemiborealen Waldzone, der südwestlichen Eichenzone von Finnland, nur aus Parainen (ca $20 \mathrm{~km} \mathrm{~S}$ von Turku) bekannt. Sie wurde an der Typusfundstelle auch am 29.VII.1986 gesammelt (3 Fruchtkörper, J. Vauras 2094 (TUR)). Der andere Fundplatz in Parainen ist in Ersby, $3.5 \mathrm{~km}$ SW von der Typuslokalität (Grid 


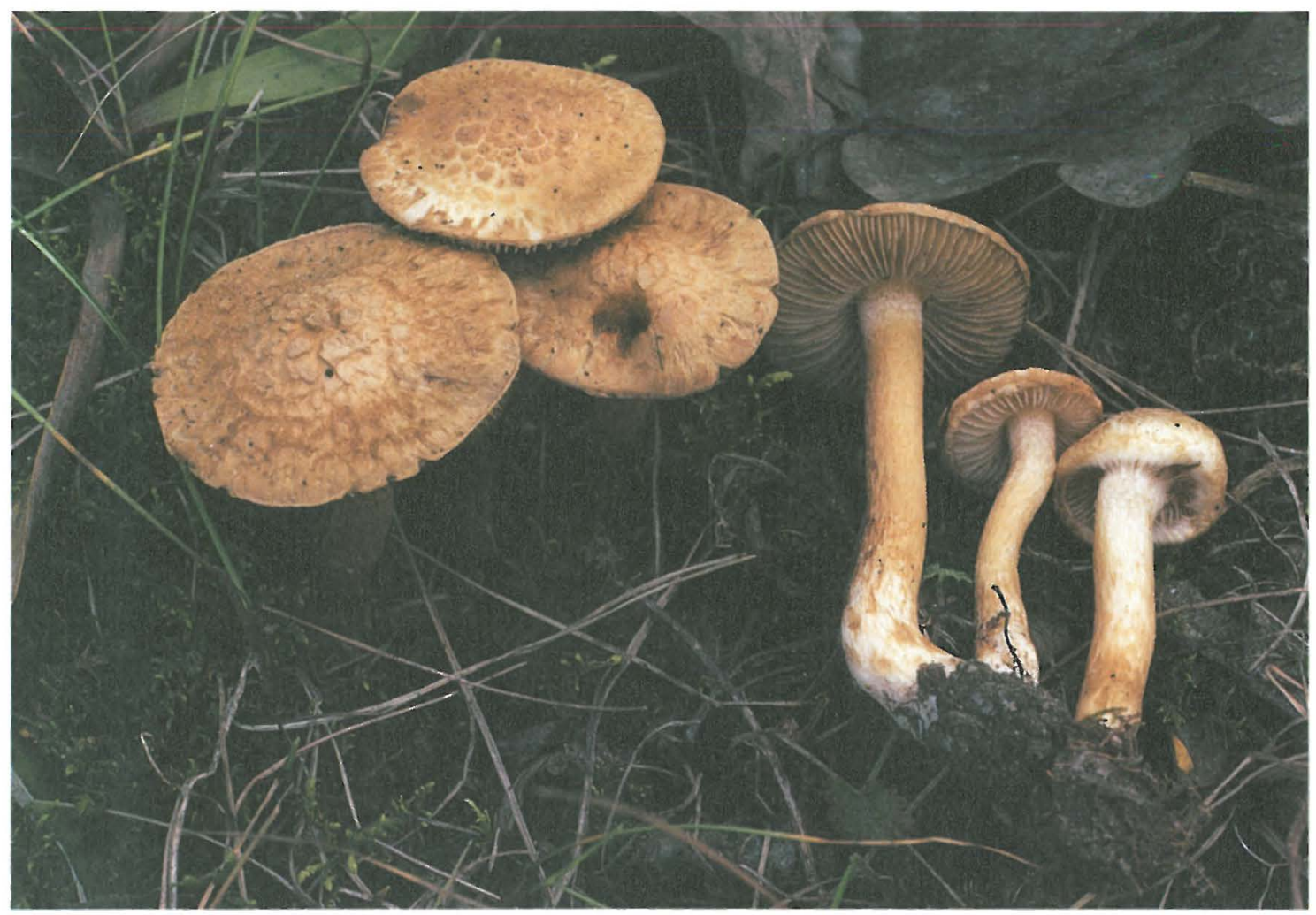

Abb. 3. Inocybe mytiliodora frisch in situ, $\times 2$ (ein Teil vom Typus). - Photo: Jukka Vauras.

27º: 66951:2367), im kurzen Gras bei Betula pendula, 1.VII.1981 J. Vauras 1007 (M, TUR). Einige pilzfloristische Untersuchungen von Parainen sind früher von Huhtinen \& Vauras (1984) und Vauras \& Huhtinen (1986) vorgestellt worden.

Der arteigene Geruch (wie bei Muscheln), die auffälligen Hymenialzystiden und der nicht bereifte Stiel haben uns neben der makroskopischen, gesamt Erscheinung (z.B. die Kleinheit) veranlasst I. mytiliodora als neue Risspilzart vorzustellen. Eine Zugehörigkeit zu zwei mit Fischgeruch beschriebenen Risspilzarten kann ausgeschlossen werden. I. pisciodora Donadini \& Riousset (I. bongardii var. pisciodora (Donadini \& Riousset) Kuyp.) gehört zur Sektion Cervicolores Sing., also zu den Risspilzarten ohne metuloide Hymenialzystiden. I. pedemontana Alessio (nom. inval.; Alessio 1980 (Art. 37.1), Alessio 1985 (Art. 45.1)) ist trennbar von I. mytiliodora durch ihren Fischgeruch und die metuloiden Kaulozystiden am oberen Teil des Stieles.

Kuyper (1986) synonymisiert I. piricystis J. Favre, I. ovoideicystis Métrod (nom. inval.) und $I$. pedemontana mit $I$. appendiculata Kühner. Nach autentischen Zeichnungen von J. Favre (in G), die wir gesehen haben, dürfte $I$. piricystis ein Synonym von I. appendiculata sein, denn auf einigen Fruchtkörper sind die Randbehänge gut zu sehen. I. mytiliodora hat eine normale Cortina, I. appendiculata einen behangene, zahnigen Hutrand. Dazu hat die letztere einen anderen Geruch, etwas grössere Sporen und durchschnittlich etwas längere und mehr dickwandige Zystiden als I. mytiliodora. I. appendiculata ist in Finnland sehr selten, wächst aber reichlich in einem Gebiet in Süd-Finnland, Varsinais-Suomi, Lohja Gemeinde, Virkkala, in der Nähe von einem Kalkwerk.

Im System Singers (1975) kann I. mytiliodora zur Sektion Inocibium, Stirps 2 zugeordnet werden. In der Aufschlüsselung nach Moser (1983) gehört sie zur Gruppe 9: "Stiel nicht bereift und Stielspitze nicht violettlich-bläulich". 


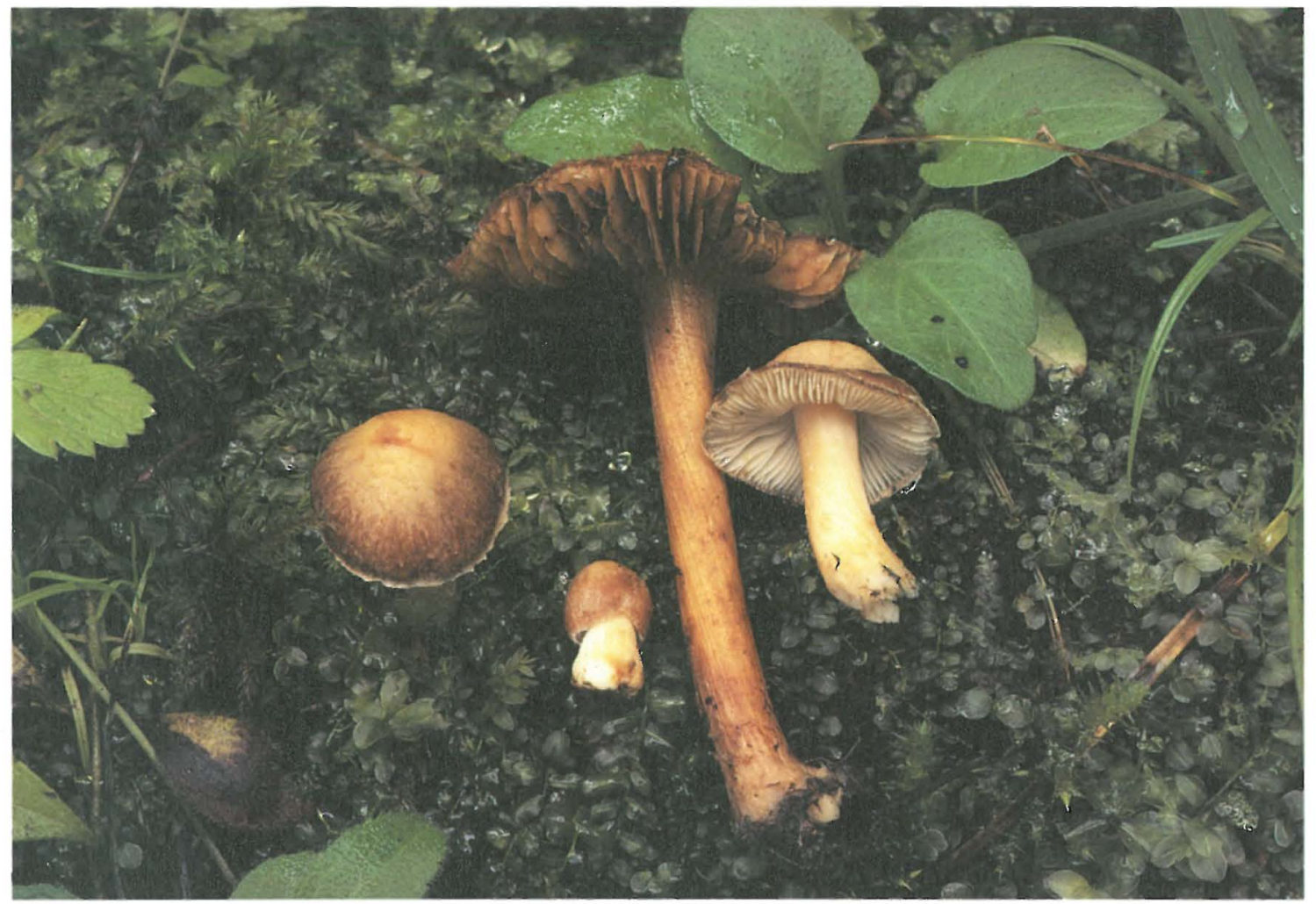

Abb. 4. Inocybe urceolicystis frisch in situ, x 1.4 (Typus). — Photo: Jukka Vauras.

Inocybe urceolicystis Stangl \& Vauras sp. nov. - Abb. 2, 4

Pileus $0.8-3.5 \mathrm{~cm}$ latus, $0.8-1 \mathrm{~cm}$ altus, primum semiglobosus, demum campanulato-convexus dein expanso-mammosus, umbone obtuso, superficies pilei sub lense paulum tomentosa, dein squamulosa, bicolor, umbone brunneo-cremeo, marginem versus brunneus. Cortina fugaci. Lamellae albae, brunneae evadentes, usque ad $6 \mathrm{~mm}$ latae. Stipes $1-5 \mathrm{~cm}$ longus, 3-5 mm crassus, cylindraceus, ad apicem pruinosus, basem versus inflatus sed non marginatus, pallide luteus, dein luteo-brunneus. Contextus pilei subalbus, in stipitem luteo-albus, brunneo-albus. Odore spermatico.

Sporae 8.5-9.5(-11) $\times(5-) 5.5-6(-6.5) \mu \mathrm{m}$, subamygdaliformes. Cystidia hymenii (40-)45-68(-80) $x$ 15-25(-30) $\mu \mathrm{m}$, ad basem rotunda, membranis in collo usque ad $5 \mu \mathrm{m}$ crassis et in $\mathrm{NH}_{4} \mathrm{OH}$ nonnihil luteis. Caulocystidia 34-85 × 12-25 um, ad basem rotunda. Habitatio: in silva inter muscos in propinquitatem Picearum et Alnorum.

Typus: Finnland. Pohjois-Savo: Kuopio, Vuorilampi Schutzgebiet, am Fussweg ca $60 \mathrm{~m} \mathrm{~N}$ von
Kolmisoppi, im Wald (Oxalis-Maianthemum-Typus) in der Nähe von Picea abies und Alnus incana, 4 Fruchtkörper, in Gesellschaft von Actaea spicata, Carex digitata, Equisetum pratense, Fragaria vesca, Geranium sylvaticum, Lonicera xylosteum, Maianthemum bifolium, Oxalis acetosella, Prunella vulgaris, Rubus saxatilis, Silene dioica, Rhytidiadelphus triquetrus, Helvella hyperborea Harmaja, Inocybe nematoloma Joss. und I. nitidiuscula (Britz.) Sacc. Der Fels ist hier basisch Lava (Hublin 1982); Grid 27ํ: 69747:5306, Höhe 95 m, 23.VIII.1981 J. Vauras 1109F (KUO, Holotypus; M, Isotypus).

Etymologie: urceolicystis, die Hymenialzystiden haben die Form des antiken Kruges.

Hut $0.8-3.5 \mathrm{~cm}$ breit, $0.8-1 \mathrm{~cm}$ hoch; jung halbkugelig, bald konisch gewölbt, alt verflachend, mit breitem wenig erhabenem Buckel; Scheitel weissgelb, braungelb (als Exsiccate Cailleux 60/N, 60/P, 67/N), Rand braun (als Exsiccate 60/P, 67/P); Hutbedeckung filzig, alt bis kleinschuppig werdend. Cortina weisslich und flüchtig. Lamellen normal, untermischt, bis $6 \mathrm{~mm}$ breit, etwas ausgerandet angewachsen; jung weiss, alt graubraun (als Exsiccate 


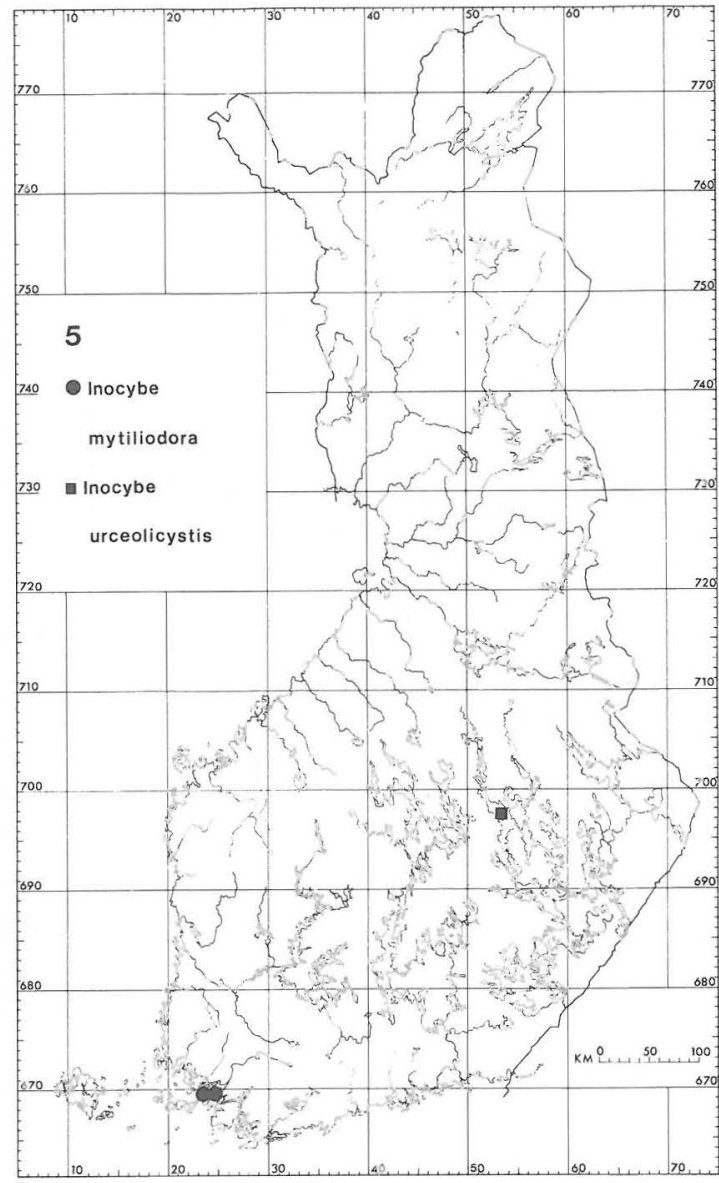

Abb. 5. Inocybe mytiliodora und Inocybe urceolicystis in Finnland.

zwischen 60 und 65/P). Stiel $1-5 \mathrm{~cm}$ hoch, 3-5 mm breit; zylindrisch, gleichdick oder zum Grund hin schwach verdickt, mit verdickter oder knolliger Basis; nur oben bereift, zur Basis hin fein befasert; jung hell gelblich, alt gelbbraun (als Exsiccate 60/P). Fleisch im Hut weisslich, im Stiel rohweisslich, Fichtenholzfarben. Geruch säuerlich, spermatisch.

Sporen (im Lamellenpräparat gemessen) 8.59.5(-11) x (5-)5.5-6(-6.5) $\mu \mathrm{m}$, Mittelwert $9.4 \times$ $5.8 \mu \mathrm{m}, \mathrm{Q}=1.5-1.7(-1.8), \overline{\mathrm{Q}}=1.6$, glatt, \pm mandelförmig. Basidien 25-32 × 10-12 $\mu \mathrm{m}$, vorwiegend mit vier Sterigmen. Hymenialzystiden (40-)45-68 $(-80) \times 15-25(-30) \mu \mathrm{m}$, im Hals mit bis zu $5 \mu \mathrm{m}$ dicken Wänden, die sich in $\mathrm{NH}_{4} \mathrm{OH}$ etwas gelb färben, bisweilen völlig gelbbraun; an Basis eigenartig abgerundet. Pleuro- und Cheilozystiden unterscheiden sich kaum voneinander. Kaulozystiden 34 $85 \times 12-25 \mu \mathrm{m}$, im Hals mit bis zu $4 \mu \mathrm{m}$ dicken
Wänden, an Basis abgerundet. Hyphen der Hutbedeckung 8-20 $\mu \mathrm{m}$ breit, deutlich angelagert inkrustiert.

Obwohl Inocybe urceolicystis, trotz vergeblicher Nachsuche, nur einmal gefunden wurde, zeigen manche Merkmalen, dass sie eine eigene Art ist. Vor allem sind uns die abgerundeten Basen der auffällig dickwandigen Hymenialzystiden sofort aufgefallen. Diese Merkmal wurde auch bei den, nur oben am Stiel anwesenden, Kaulozystiden beobachtet. Aber auch die makroskopische Gesamterscheinung, z.B. die jung hellgelbliche bis gelbbräunliche Farbe am Hutscheitel und die jung gelbliche, alt gelbbräunliche Stielfarbe (ohne jede rötliche Behauchung) sind auffällige Kennzeichnen.

Unsere Meinung ist, dass das Merkmal "Zystiden an Basis abgerundet" erblich bestimmt ist, wie leicht bei einigen eckigsporigen Risspilzen in der Sektion Petiginosae Heim (z.B. I. nematoloma, I. petiginosa (Fr.: Fr.) Gillet) zu beobachten ist. Die einzige glattsporige Risspilzart mit abgerundeten Basen der Zystiden, die uns bekannt ist, ist $I$. neomikrospora $\mathrm{Y}$. Kobay. Diese Art ist ein kleiner Pilz, der ungewöhnlich kleine Sporen hat (Kobayasi 1952).

Offensichtlich waren die Wachstumverhältnissen am Standort der I. urceolicystis, im Fundjahr 1981, aussergewöhnlich günstig, denn am selben Tage wurde auch I. nematoloma gesammelt, die auch nicht, an dieser Stelle, später wieder gefunden wurde.

I. urceolicystis gehört zur Sektion Tardae Bon (Bon 1979), wo sie mit ihren Merkmalen deutlich Artrang verdient. Der einzige Fundort ist in der südboreale Waldzone.

Danksagung. Für Hinweise zu einigen Teilen des Manuskripts danken wir Prof. Dr. Teuvo Ahti (Helsinki), Herrn Seppo Huhtinen (Turku), Dr. Thomas W. Kuyper (Leiden), Prof. Dr. Yrjö Mäkinen (Turku) und Dr. Tuomo Niemelä (Helsinki). Dr. Olivier Monthoux (Geneve) hat die autentischen Zeichnungen von J. Favre der Art Inocybe piricystis von $\mathrm{G}$ zu uns gesandt. Suomen Kulttuurirahasto und Emil Aaltonen Stiftung haben diese Arbeit finanziell unterstützt. Kuopion Luonnon Ystäväin Yhdistys hat das Herausgebung der Farbbildern mit Stipendium unterstützt. Für diese Hilfen danken wir sehr.

\section{Literatur}

Alessio, C.L. 1980: Inocybe. - Iconographia Mycologica Ab Dr. J. Bresadola 29, Suppl. 3. 367 pp. Trento.

- 1985: Complemento allo studio del genere Inocybe: $6^{\circ}$ contributo. - Boll. G. M. Bres. 28: 251-262.

Bon, M. 1979: Inocybes rares, critiques ou nouveaux dans le Nord de la France. - Beih. Sydowia 8: 76-97.

Cailleux, A. [Erscheinungsjahr nicht ausgedruckt]: Code des couleurs des sols. - Boubée. 
Hublin, P. 1982: Kuopion luonto-opas. — 44 pp. Kuopio.

Huhtinen, S. \& Vauras, J. 1984: Sienilöytöjä Paraisilta. - Sienilehti 36: 51-56.

Kobayasi, Y. 1952: On the genus Inocybe from Japan. Nagaoa 2: 76-115.

Kuyper, Th.W. 1986: A revision of the genus Inocybe in Europe 1. Subgenus Inosperma and the smooth-spored species of subgenus Inocybe. - Persoonia Suppl. 3: 1-247.

Moser, M. 1983: Die Röhrlinge und Blätterpilze. - In: Gams, H. (Begr.), Kleine Kryptogamenflora IIb/2. 533 pp. Stuttgart.

Singer, R. 1975: The Agaricales in modern taxonomy. 912 pp., 84 pl. Vaduz.

Vauras, J. \& Huhtinen, S. 1986: Finnish records on the genus Inocybe. The ecology and distribution of four calciphilous species. - Karstenia 26: 65-72.

Angenommen zur Publikation

10. November 1986 